\title{
Analysis of longitudinal vibration acceleration based on continuous time-varying model of high-speed elevator lifting system with random parameters
}

\author{
Qing Zhang ${ }^{*}$, Tao Hou, Hao Jing, and Ruijun Zhang \\ School of Mechanical and Electrical Engineering, Shandong Jianzhu University, Jinan 250101, Shandong Province, PR China
}

Received: 4 June 2019 / Accepted: 1 March 2021

\begin{abstract}
In this paper, for studying the influence of the randomness of structural parameters of high-speed elevator lifting system (HELS) caused by manufacturing error and installation error, a continuous time-varying model of HELS was constructed, considering the compensation rope mass and the tension of the tensioning system. The Galerkin weighted residual method is employed to transform the partial differential equation with infinite degrees of freedom (DOF) into the ordinary differential equation. The five-order polynomial is used to fit the actual operation state curve of elevator, and input as operation parameters. The precise integration method of time-varying model of HELS is proposed. The determination part and the random part response expression of the longitudinal dynamic response of HELS are derived by the random perturbation method. Using the precise integration method, the sensitivity of random parameters is determined by solving the random part response expression of time-varying model of HELS, and the digital characteristics of the acceleration response are analyzed. It is found that the line density of the hoisting wire rope has the maximum sensitivity on longitudinal vibration velocity response, displacement response and acceleration response, and the sensitivity of the elastic modulus of the wire rope is smallest.
\end{abstract}

Keywords: High-speed elevator lifting system / time-varying / random parameters / longitudinal vibration / acceleration response

\section{Introduction}

As a "vertically moving car", the elevator has been widely used in high-rise buildings and super high-rise buildings. In developed countries, the number of people taking elevators per day is more than that of other means of transportation, and elevators have become one of the symbols to measure the degree of modernization of the country. Withthe development of elevator toward high speed and large strokes, various vibration phenomena inevitably appear in elevators, and a large part of themis related to the elevator's lifting system.

Manufacturing error and installation error in the highspeed elevator lifting system are objective. The random parameters such as wire rope density, and elastic modulus existing in the lifting system cause the vibration of the HELS to be random vibration. The random vibration system not only affects the eigenvalues and eigenvectors of the various modes of the system, but also affects the statistical characteristics of the response [1]. In addition, studies have shown that when the initial conditions are consistent, the longitudinal vibration of the lifting system has a much greater impact on the system than the lateral

\footnotetext{
* e-mail: zhangqing@sdjzu.edu.cn
}

vibration. Therefore, it is of great significance to study the dynamic response of the longitudinal vibration random parameters of the HELS on the elevator car vibration reduction, random parameter sensitivity analysis, and safety assessment.

At present, the research on HELS mainly focuses on the dynamic characteristics of deterministic parameters [2-7]. It is rare to consider the random parameters of HELS $[1,8-10]$. The research on the random parameters of longitudinal vibration of HELS is rarer. Lin et al. [11] established elevator virtual prototype model through Solid Works, and analyzed the dynamics of the high-speed elevator car with the ADAMS, then the dynamic model of i-DOF of the elevator system in the vertical direction was established, and the sensitivity analysis is used to optimize the elevator dynamic parameters. Feng et al. [12] considered the time-varying characteristics of the elevator traction rope stiffness, established an elevator dynamics model with 8 DOF coupled vibrationand performed modal analysis on the system, according to the relationship between the natural frequency of the dynamic structure system and the excitation frequency difference, the failure mode of the system resonance is defined, and the reliability sensitivity analysis was performed on the random variables 
of the system. Wu et al. [4] used virtual prototyping technology to analyze and simulate the elevator operation dynamically, the 11 DOF vertical vibration model of the elevator system was established, through the sensitivity analysis of the high-speed elevator vibration signal, the influence of the main dynamic parameters on the highspeed elevator vibration was obtained. Although the above literatures consider random parameters for the study of longitudinal vibration of elevators, its research is based on the discrete model of elevator concentration parameters, but its research is based on the elevator lumped parameter model. The ordinary differential control equations established by this type of model are simple, easy to understand, andsolve. However, because such models ignore the continuous characteristics of the wire rope, they cannot better reflect the dynamic characteristics of the elevator lifting system.

The establishment of the distributed parameter model of the HELS draws on the research theory of the axially moving string, which is simplified into a section of axial motion string with concentrated mass, which can better describe the flexible time-varying characteristics of the traction wire rope, so it is gradually being applied. Zhang et al. [13] simplified the elevator hoisting rope to a variable length axial motion string with a certain mass attached to one end, the differential equations and energy equations for the vertical vibration of the HELS were established by the energy method and the Hamilton principle. Bao et al. [2,14] used the Hamliton principle to construct a lateral vibration control equation for flexible wire rope without external excitation and external excitation, and evaluated the theoretical model through experiments, the experimental results well agree with the theoretical predictions. In addition [15], considering the interaction between the rigid motion and the deformation motion of the steel wire rope, the differential equation of the wire rope motion of the lifting system is constructed, and the model is analyzed. However, the above literature does not consider the effect of the compensation rope mass and the tension of the tensioning system on the vibration of the HELS.

Therefore, under the premise of comprehensively considering the influence of compensation rope mass and tension of the tensioning system, the time-varying continuous model of HELS is constructed by combining energy method and Hamilton principle. Using random perturbation method to derive the dynamic equation of system response under random parameters. Applying the precise integration method of HELS to analyze the sensitivity and standard deviation of structural random parameters of elevator operation process. Then the influences of each structural parameters on the dynamic characteristics of the lifting system are analyzed.

\section{Establishment of longitudinal time-varying model for high-speed traction elevator lifting system}

To study the time-varying characteristics of the longitudinal vibration of traction rope conveniently, the modeling and solution of this paper are based on the following three assumptions:

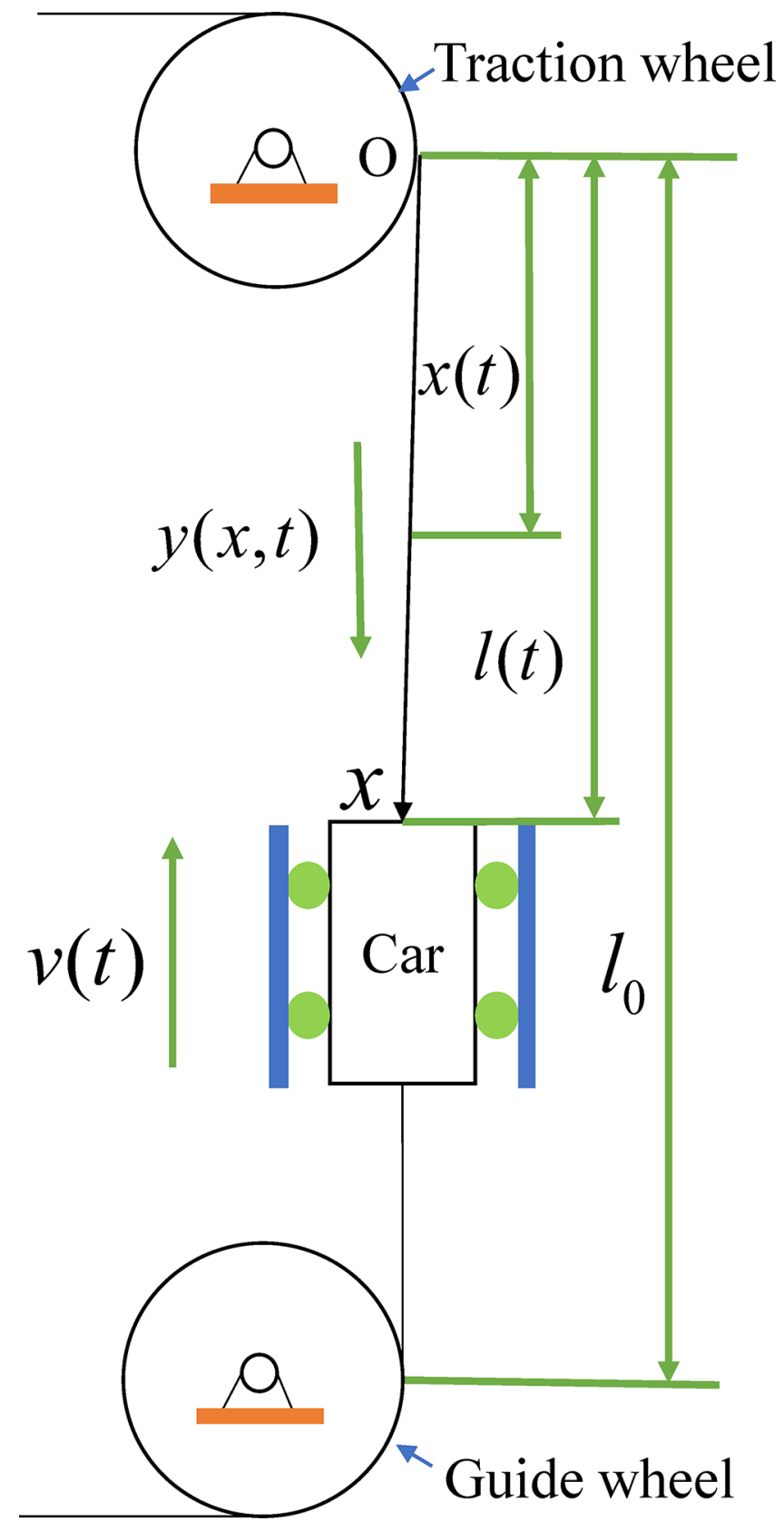

Fig. 1. Time-varying model of the hoisting rope in an elevator lifting system.

- Hoisting ropes are continuous and uniform, with constant cross-sectional area $A$ and elastic modulus $E$ during movement;

- The influence of lateral vibration from the hoisting ropes is ignored, and elastic deformation caused by the vertical vibration of the hoisting ropes is smaller than the length of the ropes;

- The influences of bending rigidity on hoisting ropes, friction force, and airflow are ignored.

Figure 1 shows the time-varying model of longitudinal vibration in a high-speed traction elevator lifting system. The hoisting rope of the high-speed traction elevator is 
simplified as a variable-length string along the axial force and movement. The specified structure of the car is ignored and the structure is simplified into a rigid weight block of mass $m$ connected to the lower end of the cord. $\rho_{1}$ is the density of the hoisting rope, $A$ is the cross-sectional area, $E$ is the elastic modulus, and $\rho_{2}$ is the density of compensation rope. The origin of the coordinates is the tangent point of the traction sheave and the hoisting rope, and the direction vertically downward is the positive direction of the $\mathrm{X}$ axis. The length of hoisting rope at the top of the car from the origin of the coordinate is $l(t)$. The vibration displacement at the string $x(t)$ is $y(x(t), t)$, and $v(t)$ is the operating speed of the high-speed traction elevator. $l_{0}$ is the maximum lift height (high-speed traction elevators are generally used in super high-rise buildings. The height of the car is very small compared to the super high-rise buildings, so the height of the car is negligible).

By using the finite deformation theory of continuum, the displacement vector and velocity vector of $x(t)$ in the $\mathrm{X}$-axis are as follows:

$$
\begin{gathered}
r=[x(t)+y(x(t), t)] j, \\
V=\left[v(t)+y_{t}(x(t), t)\right] j,
\end{gathered}
$$

where $j$ is the unit vector in the $\mathrm{X}$-axis direction, $y(x(t), t)$ and $y_{t}(x(t), t)$ are the partial derivatives of $t$, and $y, y_{t}$ represent $y(x(t), t)$ and $y_{t}(x(t), t)$, respectively.

Similarly, the displacement vector and velocity vector of the car in the direction of $\mathrm{X}$-axis are as follows:

$$
\begin{gathered}
r_{c}=[l(t)+y] j, \\
V_{c}=\left[v(t)+y_{t}\right] j,
\end{gathered}
$$

The kinetic energy of the system can be expressed as follows:

$$
E_{k}=\left.\frac{1}{2} m V^{2}\right|_{x=l(t)}+\frac{1}{2} \rho_{1} \int_{0}^{l(t)} V^{2} d s,
$$

The elastic potential of the system is:

$$
E_{s}=\int_{0}^{l(t)}\left(P y_{x}+\frac{1}{2} E A y_{x}^{2}\right) d s,
$$

where $y_{x}(x(t), t)$ is the partial derivative of $x$, and $y_{x}$ represents $y_{x}(x(t), t)$.

$P$ is the tension of the rope during a static balance of tension. While the hoisting rope is subjected to its own gravity and the gravity of the car, it is also subjected to the gravity of the tensioning rope and the pre-tensioning force $f$ of the tensioning device. Thus, tension $P$ in the static balance can be expressed as:

$$
P=\left[m+\rho_{1}(l(t)-x)+\rho_{2}\left(l_{0}-l(t)\right)\right] g+f,
$$

The gravitational potential energy of the system is expressed as:

$$
E_{g}=-\int_{0}^{l(t)} \rho_{1} g d t-\left.m g y\right|_{x=l(t)},
$$

According to the Hamilton principle:

$$
I=\int_{t_{1}}^{t_{2}}\left[\delta E_{k}-\delta E_{s}-\delta E_{g}\right] d t=0,
$$

The longitudinal vibration dynamics equation of the high-speed traction elevator lifting system arederived as follows:

$$
\begin{gathered}
\rho_{1}\left(y_{t t}+a\right)-P_{x}-\rho_{1} g-E A y_{x x}=0,(0<x<l(t)),(10) \\
m\left(a+y_{t t}\right)+\rho_{1} v\left(v+y_{t}\right)+E A y_{x}+P-m g=0,(x=l(t)),
\end{gathered}
$$

Equation (11) is the boundary condition where the string is at $x=l(t)$.

\section{Galerkin discretization of time-varying partial differential equations for the HELS}

The algebraic equation coefficient matrix obtained by Galerkin discrete method is symmetric, and approximation accuracy is higher than those of the other methods. Therefore, the Galerkin method is used to discretize the partial differential control equation.

For facilitating the discrete method, a dimensionless parameter $\xi$ is introduced, and normalize the original variables, that is, $\xi=x / l(t)$. The time domain of $x$ becomes the fixed domain $[0,1]$ of $\xi$. Assuming that the solution of equation (10) can be represented by infinite DOF distribution function $y$ :

$$
y(x, t)=\sum_{i=1}^{n} \varphi_{i}(\xi) q_{i}(t)=\sum_{i=1}^{n} \varphi_{i}\left(\frac{x}{l(t)}\right) q_{i}(t),
$$

$\varphi_{i}(\xi)$ is the trial function, and $q_{i}(t)$ is the timedependent generalized coordinates

$$
\varphi_{i}(\xi)=\sqrt{2} \sin \left(\frac{2 i-1}{2} \pi \xi\right)(i=1,2, \ldots, n),
$$

Then,

$$
\begin{aligned}
y_{x}= & \frac{1}{l(t)} \sum_{i=1}^{n} \varphi_{i}^{\prime}(\xi) q_{i}(t), y_{x x}=\frac{1}{l(t)} \sum_{i=1}^{n} \varphi_{i}^{\prime \prime}(\xi) q_{i}(t) \\
y_{t}= & \sum_{i=1}^{n} \varphi_{i}(\xi) \dot{q}_{i}(t)-\frac{\xi v}{l(t)} \sum_{i=1}^{n} \varphi_{i}^{\prime}(\xi) q_{i}(t) \\
y_{t t}= & \sum_{i=1}^{n} \varphi_{i}(\xi) \ddot{q}_{i}(t)-\frac{2 \xi v}{l(t)} \sum_{i=1}^{n} \varphi_{i}^{\prime}(\xi) \dot{q}_{i}(t)+\frac{2 \xi v^{2}}{l^{2}(t)} \sum_{i=1}^{n} \varphi_{i}^{\prime}(\xi) q_{i}(t) \\
& -\frac{a \xi}{l(t)} \sum_{i=1}^{n} \varphi_{i}{ }_{i}(\xi) q_{i}(t)+\frac{\xi^{2} v^{2}}{l^{2}(t)} \sum_{i=1}^{n} \varphi_{i}^{\prime \prime}(\xi) q_{i}(t)
\end{aligned}
$$

Substitute equation (14) into the kinetic equation (11), and multiply both sides by $\varphi_{j}(\xi)$, and integrate $\xi$ in the range $[0,1]$. Substitute equation (14) into the boundary condition (12), and multiply both sides by $\varphi_{j}(1)$ after 
transformation. The original partial differential equations are discretized into the following equation by using the weighted residual method:

$$
\mathbf{M} \ddot{\boldsymbol{q}}_{j}+\mathbf{C} \dot{\boldsymbol{q}}_{j}+\mathbf{K} \mathbf{q}_{j}=\mathbf{F}
$$

where, $\mathbf{q}_{j}=\left[q_{1}(t), q_{2}(t), \ldots, q_{n}(t)\right]$, is the generalized coordinate vector, $\boldsymbol{M}, \boldsymbol{C}, \boldsymbol{K}$, and $\boldsymbol{F}$ are the mass, damping, stiffness, and generalized force matrices, respectively. And,

$$
\begin{gathered}
\mathbf{M}=\rho_{1} \delta_{I J}+\frac{m}{l} \varphi_{i}(1) \varphi_{j}(1) \\
\mathbf{C}=-\frac{2 \rho_{1} v}{l} \int_{0}^{1} \xi \varphi_{i}^{\prime} \varphi_{j} d \xi+\frac{\rho_{1} v}{l} \varphi_{i}(1) \varphi_{j}(1) \\
\boldsymbol{K}=\frac{m v^{2}}{l^{3}} \varphi_{i}^{\prime \prime}(1) \varphi_{j}(1)-\frac{\rho_{1} a}{l} \int_{0}^{1} \xi \varphi_{i}^{\prime} \varphi_{j} d \xi \\
-\frac{\rho_{1} v^{2}}{l^{2}} \int_{0}^{1} \xi^{2} \varphi_{i}^{\prime} \varphi_{j}^{\prime} d \xi-\frac{E A}{l^{2}} \int_{0}^{1} \varphi_{i}^{\prime \prime} \varphi_{j} d \xi \\
\boldsymbol{F}=-\rho_{1} a \int_{0}^{1} \varphi_{j} d \xi-\frac{m a}{l} \varphi_{j}(1)-\frac{\rho_{1} v^{2}}{l} \varphi_{j}(1) \\
-\frac{\rho_{2} g\left(l_{0}-l\right)+f}{l} \varphi_{j}(1)
\end{gathered}
$$

\section{Precise integration method for vertical vibration model of the time-varying system in the high-speed traction elevator}

For the time-varying dynamic model of the high-speed elevator traction hoisting system established above, because of its strong time-varying characteristics, the mass, damping and stiffness of the system are changing every moment. It is difficult for the general numerical method to achieve high accuracy for this kind of problem. Precise integration method, due to its explicit stability and high accuracy [16], has been widely used in solving dynamics of nonlinear time-varying systems, and achieved good results $[17,18]$. Therefore, for the high-speed elevator time-varying model established in this paper, the precise integration method of the longitudinal time-varying model of HELS is proposed to analyze the model, so as to make the result more accurate.

First, follow the introduction of the dual variable of Hamiltonian system [19],

$$
p=M \dot{x}+C(t) x / 2 \text { or } \dot{x}=M^{-1} p-M^{-1} C(t) x / 2
$$

By substituting the equation (16) into the dynamic equation, the following equation can be obtained:

$$
\dot{p}=\left(C(t) M^{-1} C(t) / 4-K(t)\right) x-C(t) M^{-1} p / 2+f(t)
$$

The above equations are written in the general form of a linear system

$$
\left\{\begin{array}{l}
\dot{x}=A x+C p+r_{x} \\
\dot{p}=B x+D p+r_{p}
\end{array}\right.
$$

where, $\quad A=-M^{-1} C(t) / 2, \quad B=C(t) M^{-1} C(t) / 4-K(t)$, $C=-C(t) M^{-1} / 2, D=M^{-1}, r_{p}=0, r_{x}=f(t)$.

Therefore,

$$
\dot{z}=H z+\phi(t)
$$

where, $z=\left[\begin{array}{l}x \\ p\end{array}\right], H=\left[\begin{array}{ll}A & D \\ B & \mathrm{C}\end{array}\right], \phi(t)=\left[\begin{array}{l}\mathrm{r}_{x} \\ r_{p}\end{array}\right]$.

Assume that the nonhomogeneous term is linear in time step $\left(t_{k}, t_{k}+1\right)$, the equation is

$$
\dot{z}=H z+\phi_{k}+\dot{\phi}_{k}\left(t-t_{k}\right)
$$

Then, the solution at $t_{k+1}$ moment can be written as

$$
\begin{aligned}
\mathrm{z}_{k+1}= & T_{k}\left[z_{k}+H_{k}{ }^{-1}\left(\phi_{k}+H_{k}{ }^{-1} \dot{\phi}_{k}\right)\right] \\
& -H_{k}{ }^{-1}\left[\phi_{k}+H_{k}{ }^{-1} \dot{\phi}_{k}+\dot{\phi}_{k}\left(t_{k+1}-t_{k}\right)\right]
\end{aligned}
$$

where, $T_{\mathrm{k}}=e^{H_{k}}\left(t_{k+1}-t_{k}\right)$.

Then the solution of the equation is transformed into the solution matrix $\boldsymbol{T}_{\boldsymbol{k}}$, and the accuracy of the matrix $\boldsymbol{T}_{\boldsymbol{k}}$ becomes the key to solving the equation. Zhong [19] proposed a $2^{N}$ algorithm by using the additive theorem. For the above formula, there is,

$$
T_{k}=e^{H_{k} \Delta t}=\left(e^{H_{k} \frac{\Delta t}{m}}\right)^{m}=\left(e^{H_{k} \tau}\right)^{m}
$$

Among them, choose $m=2^{N}$, usually, $\Delta t$ is a small time interval, so $\tau=\Delta t / m$ is a very small time interval, for $\tau$, there is,

$$
e^{H_{k} \tau} \approx I+H_{k} \cdot \tau+\frac{\left(H_{k} \cdot \tau\right)^{2}}{2}=I+T_{a}
$$

where $I$ is the identity matrix, $T_{a}=\left(\mathrm{H}_{k} \cdot \tau\right) \cdot\left(\mathrm{I}+H_{k} \cdot \tau / 2\right)$.

Therefore, the matrix $\boldsymbol{T}_{\boldsymbol{k}}$ can be decomposed as follows,

$$
T_{k}=\left(I+T_{a}\right)^{2^{N}}=\left[T_{a}+I\right]^{2^{N-1}} \times\left[T_{a}+I\right]^{2^{N-1}}
$$

According to the flow chart, as shown in Figure 2, $T_{k}=I+T_{a}$. Then, according to equation (21), given initial condition $z_{0}$, the steps are gradually performed to obtain $\mathrm{z}_{1}, z_{2}, \ldots, z_{k}, \ldots$, which is a typical "self-starting" algorithm.

\section{The quintic polynomial fitting for the running state curve of the elevator}

According to the actual operation state of the elevator, the description of the operation state stage of the elevator when it goes up is shown in Table 1 below. The fifth-order 


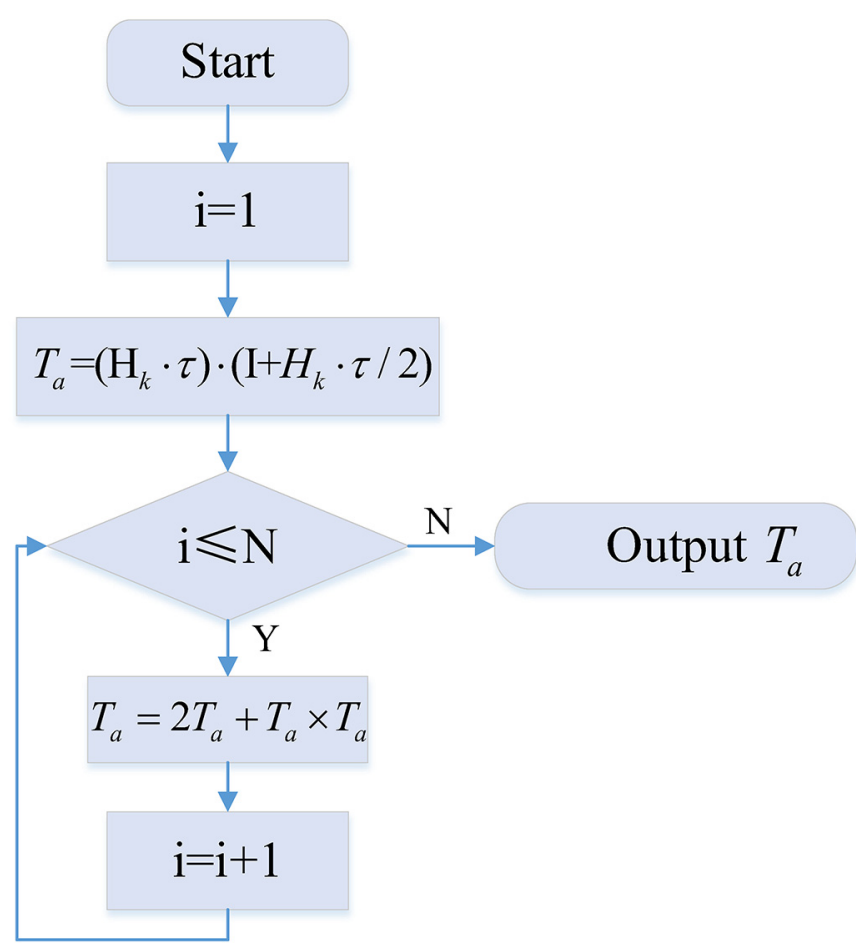

Fig. 2. Operation flow chart.

Table 1. Stage division of elevator operation state curve.

\begin{tabular}{lll}
\hline $\begin{array}{c}\text { Stage } \\
\text { Time } \\
\text { required }\end{array}$ & Stage description \\
\hline 1 & $t_{j}$ & Acceleration increases to $a=-a_{\max }$ \\
2 & $t_{a}$ & Acceleration remains $-a_{\max }$ constant \\
3 & $t_{j}$ & Acceleration decreases to zero, $v=-v_{\max }$ \\
4 & $t_{v}$ & Speed $v_{\max }$ is unchanged \\
5 & $t_{j}$ & Acceleration increases to $a=a_{\max }$ \\
6 & $t_{a}$ & Acceleration remains $a_{\max }$ constant \\
7 & $t_{j}$ & Acceleration decreases to zero, $v=0$ \\
\hline
\end{tabular}

polynomial (25) is used to fit the actual operation state of the elevator, and the operation curves of the elevator in each stage can be obtained, as shown in Figure 3 below.

$$
l_{i}(t)=C_{0}^{i}+C_{1}^{i} t+C_{2}^{i} t^{2}+C_{3}^{i} t^{3}+C_{4}^{i} t^{4}+C_{5}^{i} t^{5}
$$

\section{Sensitivity analysis of random parameters of lifting system based on random perturbation method}

The structural parameters (such as the line density of traction rope $\rho_{1}$, cross-sectional area of traction rope $A$, elastic modulus of traction rope $E$ ) of HELS have certain randomness. Therefore, $\boldsymbol{M}, \boldsymbol{C}, \boldsymbol{K}, \boldsymbol{F}$ in the differential equation of system dynamics has stochastic property. The following transformations are required.

$$
\left\{\begin{array}{l}
M=M_{d}+\varepsilon M_{r} \\
C=C_{d}+\varepsilon C_{r} \\
K=K_{d}+\varepsilon K_{r} \\
Y=Y_{d}+\varepsilon Y_{r} \\
F(t)=F_{d}(t)+\varepsilon F_{r}(t)
\end{array}\right.
$$

where $\varepsilon$ is a small parameter [20,21]. The subscripts $d$ and $r$ respectively represent the determined part and the random part of the random variable.

Substituting equation (26) into (16) and expand to compare $\varepsilon$ with the same power coefficient. Omitting higher-order terms above $O\left(\varepsilon^{2}\right)$ the following equations are obtained.

$$
\begin{gathered}
\varepsilon^{0}: M_{d} \ddot{q}_{d}+C_{d} \dot{q}_{d}+C_{d} q_{d}=F_{d}(t) \\
\varepsilon^{1}: M_{d} \ddot{q}_{r}+C_{d} \dot{q}_{r}+K_{d} q_{r} \\
=F_{r}(t)-\left(M_{r} \ddot{q}_{d}+C_{r} \dot{q}_{d}+K_{r} q_{d}\right)
\end{gathered}
$$

Equations (27) and (28) represent the deterministic part and the random part of the response, respectively. For convenience, the random response $\left\{q_{r}\right\}$ is divided into two parts:

$$
q_{r}=q_{r 1}+q_{r 2}
$$

where $\left\{q_{r 1}\right\}$ and $\left\{q_{r 2}\right\}$ respectively satisfy the following equation.

$$
\begin{gathered}
M_{d} \ddot{q}_{r 1}+C_{d} \dot{q}_{r 1}+K_{d} q_{r 1}=F_{r}(t) \\
M_{d} \ddot{q}_{r 2}+C_{d} \dot{q}_{r 2}+K_{d} q_{r 2}=-\left(M_{r} \ddot{q}_{d}+C_{r} \dot{q}_{d}+K_{r} q_{d}\right)
\end{gathered}
$$

Equations (30) and (31) represent random responses due to randomness of excitation and randomness of parameters, respectively. For equation (31), the random variable can be Taylor-expanded near the determined portion $b_{d j}(j=1,2, \ldots m)$ of the random parameter [22,23].

$$
\begin{gathered}
\ddot{q}_{r 2}=\sum_{j=1}^{m} \frac{\partial \ddot{q}_{d}}{\partial b_{j}} \cdot b_{r j} \\
\dot{q}_{r 2}=\sum_{j=1}^{m} \frac{\partial \dot{q}_{d}}{\partial b_{j}} \cdot b_{r j} \\
q_{r 2}=\sum_{j=1}^{m} \frac{\partial q_{d}}{\partial b_{j}} \cdot b_{r j} \\
M_{r}=\sum_{j=1}^{m} \frac{\partial M_{d}}{\partial b_{j}} \cdot b_{r j}
\end{gathered}
$$



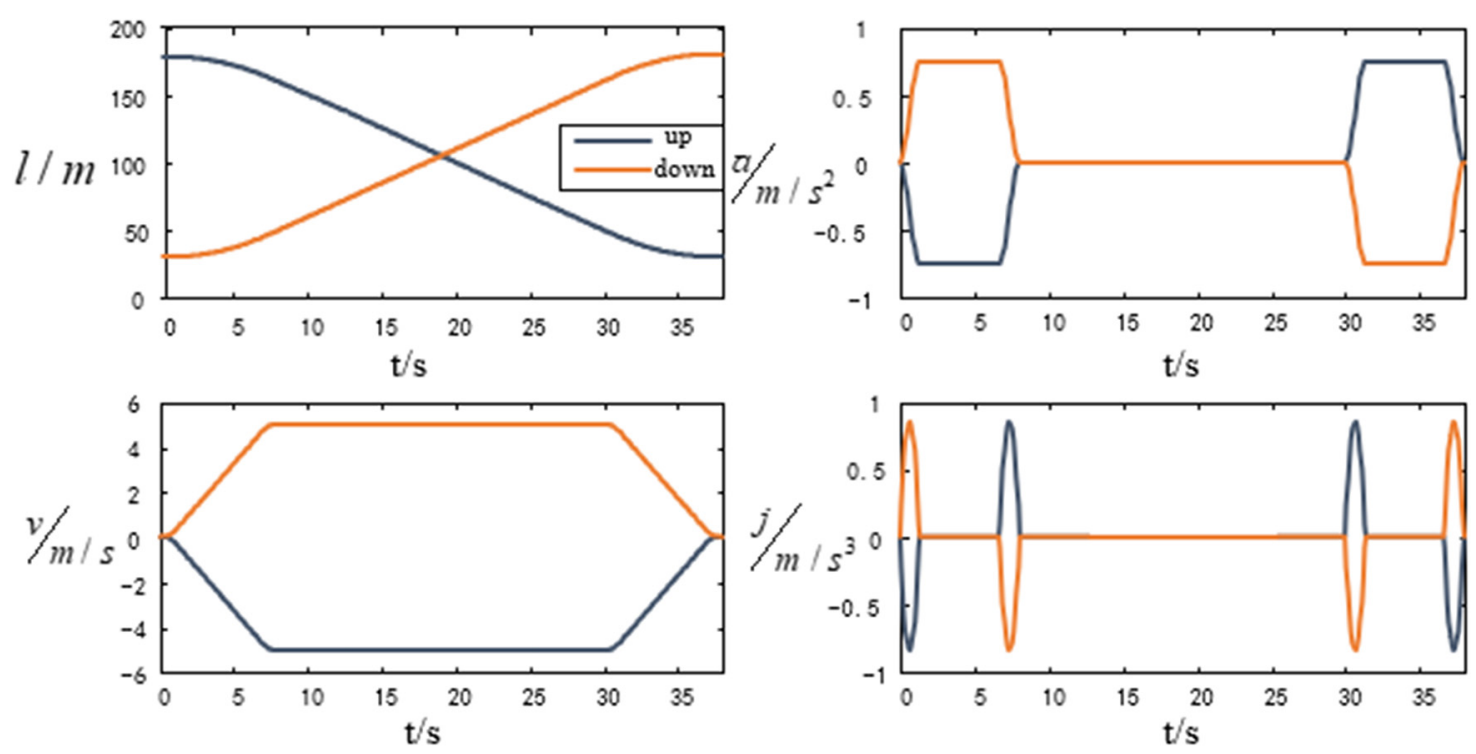

Fig. 3. Elevator running state curve.

$$
\begin{gathered}
C_{r}=\sum_{j=1}^{m} \frac{\partial C_{d}}{\partial b_{j}} \cdot b_{r j} \\
K_{r}=\sum_{j=1}^{m} \frac{\partial K_{d}}{\partial b_{j}} \cdot b_{r j}
\end{gathered}
$$

Substituting equations (31)-(37) into equation (31) and comparing the coefficients of $b_{r j}$

$$
\begin{gathered}
M_{d} \frac{\partial \ddot{q}_{d}}{\partial b_{j}}+C_{d} \frac{\partial \dot{q}_{d}}{\partial b_{j}}+K_{d} \frac{\partial q_{d}}{\partial b_{j}}=-\left(\frac{\partial M_{d}}{\partial b_{j}} \ddot{q}_{d}+\frac{\partial C_{d}}{\partial b_{j}} \dot{q}_{d}+\frac{\partial K_{d}}{\partial b_{j}} q_{d}\right) \\
\times(j=1,2, \ldots, m)
\end{gathered}
$$

Using the precise integration method for vertical vibration model of the time-varying system in the highspeed traction elevator to solve the equation (38), the sensitivity of the system response, $\frac{\partial \ddot{\boldsymbol{q}}_{d}}{\partial b_{j}}, \frac{\partial \dot{\boldsymbol{q}}_{d}}{\partial b_{j}}$, and $\frac{\partial \boldsymbol{q}_{d}}{\partial b_{j}}$ can be get.

\section{Analysis of mean and standard deviation of longitudinal vibration acceleration of high- speed elevators with random parameters}

Define the covariance matrix of the displacement response of the continuous time-varying model as $\mathbf{N}_{\mathbf{q}}$, the random parameter covariance matrix as $\mathbf{N}_{b}$, and the displacement response sensitivity matrix as $\left[\frac{\partial \boldsymbol{q}_{d}}{\partial_{b}}\right]$.
$N_{q}=$

$\times\left[\begin{array}{cccc}\operatorname{Var}\left(q^{(1)}\right) & \operatorname{Cov}\left(q^{(2)}, q^{(1)}\right) & \ldots & \operatorname{Cov}\left(q^{(k)}, q^{(1)}\right) \\ \operatorname{Cov}\left(q^{(2)}, q^{(1)}\right) & \operatorname{Var}\left(q^{(1)}\right) & \ldots & \operatorname{Cov}\left(q^{(k)}, q^{(2)}\right) \\ \vdots & \vdots & \ddots & \vdots \\ \operatorname{Cov}\left(q^{(k)}, q^{(1)}\right) & \operatorname{Cov}\left(q^{(k)}, q^{(2)}\right) & \ldots & \operatorname{Var}\left(q^{(k)}\right)\end{array}\right]$

$$
\begin{gathered}
N_{b}=\left[\begin{array}{cccc}
\operatorname{Var}\left(b_{1}\right) & \operatorname{Cov}\left(b_{2}, b_{1}\right) & \ldots & \operatorname{Cov}\left(b_{m}, b_{1}\right) \\
\operatorname{Cov}\left(b_{2}, b_{1}\right) & \operatorname{Var}\left(b_{2}\right) & \ldots & \operatorname{Cov}\left(b_{m}, b_{2}\right) \\
\vdots & \vdots & \ddots & \vdots \\
\operatorname{Cov}\left(b_{m}, b_{1}\right) & \operatorname{Cov}\left(b_{m}, b_{2}\right) & \ldots & \operatorname{Var}\left(b_{m}\right)
\end{array}\right] \\
{\left[\frac{\partial \boldsymbol{q}_{d}}{\partial b}\right]=\left[\begin{array}{llll}
\frac{\partial \boldsymbol{q}_{d}}{\partial b_{1}} & \frac{\partial \boldsymbol{q}_{d}}{\partial b_{2}} & \ldots & \frac{\partial \boldsymbol{q}_{d}}{\partial b_{m}}
\end{array}\right]}
\end{gathered}
$$

where $\operatorname{Var}\left(q^{(k)}\right)$ represents the variance of the $k_{t h}$ element in the vector $\boldsymbol{q}$, and Cov represents the covariance.

$$
\boldsymbol{N}_{q}=\left[\frac{\partial \boldsymbol{q}_{d}}{\partial b}\right] \boldsymbol{N}_{b}\left[\frac{\partial \boldsymbol{q}_{d}}{\partial b}\right]^{T}
$$

The standard deviation of displacement response can be obtained by solving equation (36).

$$
\sigma_{q}^{i}=\left(\sum_{j=1}^{m} \sum_{k=1}^{m} \frac{\partial \boldsymbol{q}_{d}^{i}}{\partial b_{j}} \frac{\partial \boldsymbol{q}_{d}^{i}}{\partial b_{k}} \sigma_{b j} \sigma_{b k} \rho_{j k}\right)^{1 / 2}
$$


Table 2. HELS random parameter value.

\begin{tabular}{lll}
\hline Variable $b_{j}$ & Mean $\mathrm{D}_{b j}$ & $\begin{array}{l}\text { Standard deviation } \sigma_{b j} \\
(\mathrm{CV}=0.02)\end{array}$ \\
\hline The lift mass $(\mathrm{m} / \mathrm{kg})$ & 400 & 8 \\
The elastic modulus of the wire rope $\left(\mathrm{N} / \mathrm{m}^{2}\right)$ & $8 \times 10^{10}$ & $16 \times 10^{8}$ \\
The wire rope density $(\mathrm{kg} / \mathrm{m})$ & 0.87 & $1.74 \times 10^{-2}$ \\
\hline
\end{tabular}

Table 3. Random parameter sensitivity mean.

\begin{tabular}{llll}
\hline Random parameter & $E\left|\frac{\partial \ddot{q}_{d}}{\partial b_{j}}\right|$ & $E\left|\frac{\partial \dot{q}_{d}}{\partial b_{j}}\right|$ & $E\left|\frac{\partial q_{d}}{\partial b_{j}}\right|$ \\
\hline The lift mass & $8.219 \times 10^{-4}$ & $4.882 \times 10^{-5}$ & $3.445 \times 10^{-6}$ \\
The elastic modulus of lifting steel wire rope & $2.172 \times 10^{-11}$ & $1.53 \times 10^{-12}$ & $1.877 \times 10^{-13}$ \\
The wire rope density & $7.376 \times 10^{-2}$ & $4.391 \times 10^{-3}$ & $3.145 \times 10^{-4}$ \\
\hline
\end{tabular}

where $\sigma_{q}^{i}$ is the standard deviation $\left[\operatorname{Var}\left(q^{(i)}\right)\right]^{1 / 2}$ of the i-th element in vector $\boldsymbol{X}, \rho_{j k}$ is the correlation coefficient between $b_{j}$ and $b_{k}$.

Similarly, the standard deviations of velocity and acceleration responses can be obtained:

$$
\begin{aligned}
\sigma_{\dot{q}}^{i} & =\left(\sum_{j=1}^{m} \sum_{k=1}^{m} \frac{\partial \dot{\boldsymbol{q}}_{d}^{i}}{\partial b_{j}} \frac{\partial \dot{\boldsymbol{q}}_{d}^{i}}{\partial b_{k}} \sigma_{b j} \sigma_{b k} \rho_{j k}\right)^{1 / 2} \\
\sigma_{\ddot{q}}^{i} & =\left(\sum_{j=1}^{m} \sum_{k=1}^{m} \frac{\partial \ddot{\boldsymbol{q}}_{d}^{i}}{\partial b_{j}} \frac{\partial \ddot{\boldsymbol{q}}_{d}^{i}}{\partial b_{k}} \sigma_{b j} \sigma_{b k} \rho_{j k}\right)^{1 / 2}
\end{aligned}
$$

\section{Case analysis}

Taking a HELS as an example. Its maximum operating speed is $v=5 \mathrm{~m} / \mathrm{s}$, the elevator operating parameters are as shown in Section 4. the elastic modulus of the wire rope is $E=8 \times 10^{10} \mathrm{~N} / \mathrm{m}^{2}$, lifting wire rope cross-sectional area $A=89.344 \mathrm{~cm}^{2}, \quad$ compensation rope line density $\rho_{2}=0.343 \mathrm{~kg} / \mathrm{m}$, tension $F=300 \mathrm{~N}$. The longitudinal time-varying model of hoisting system has independent random parameters (the lift mass, the elastic modulus of the wire rope, and the wire rope density) and obeys normal distribution. The coefficient of variation takes $\mathrm{CV}=0.02$, and each random parameter is shown in Table 2 below.

\subsection{Random parameter sensitivity analysis}

The precise integration method is employed to solve the response sensitivity equation of the random parameter system, equation (38). The vibration displacement, vibration velocity and acceleration response sensitivity of each random parameter are obtained. After taking the absolute value and calculating the mean values $E\left|\frac{\partial \ddot{q}_{d}}{\partial b_{j}}\right|, E\left|\frac{\partial \dot{q}_{d}}{\partial b_{j}}\right|$, $E\left|\frac{\partial q_{d}}{\partial b_{j}}\right|$, the calculation results are shown in Table 3.
It can be seen from Table 3 that, among the three random parameters, the linear density of the hoisting wire rope is the most sensitive to the vibration velocity response, the vibration displacement response, and the vibration acceleration response. And the three random parameters of the lift mass, the elastic modulus of the lifting steel wire rope and the wire rope density are the most sensitive to the acceleration response, followed by the vibration velocity response, and the sensitivity to the vibration displacement response is the smallest.

\subsection{Random parameter acceleration and jerk response analysis}

Using the response expression constructed by the perturbation theory, the precise integral method is used to solve the equation (38) to obtain the response acceleration $\ddot{q}_{d}$, the longitudinal vibration acceleration response of the highspeed elevator is determined as shown in Figure 4a. The longitudinal vibration jerk response is determined as shown in Figure 4b.

Take the random part of the random parameter $b_{r j}= \pm \sigma_{b j}$, and substitute it with the obtained $\ddot{q}_{d}$ into equation (38), Solve the random part of the acceleration response, and then superimpose with $\ddot{q}_{d}$ to get the total acceleration response, as shown in Figure 5a. In addition, the total jerk response as shown in Figure 5b.

Analysis the acceleration and jerk response determination part of the HELS, it can be seen that the absolute value of the longitudinal maximum acceleration and jerk increases after considering the influence of the random parameter, which is increased by about $50 \%$. The corresponding values of the longitudinal acceleration and jerk total response have different degrees of dispersion at each moment, indicating that the dispersion of the longitudinal acceleration and jerk response is increased after considering the randomness of the parameters. 


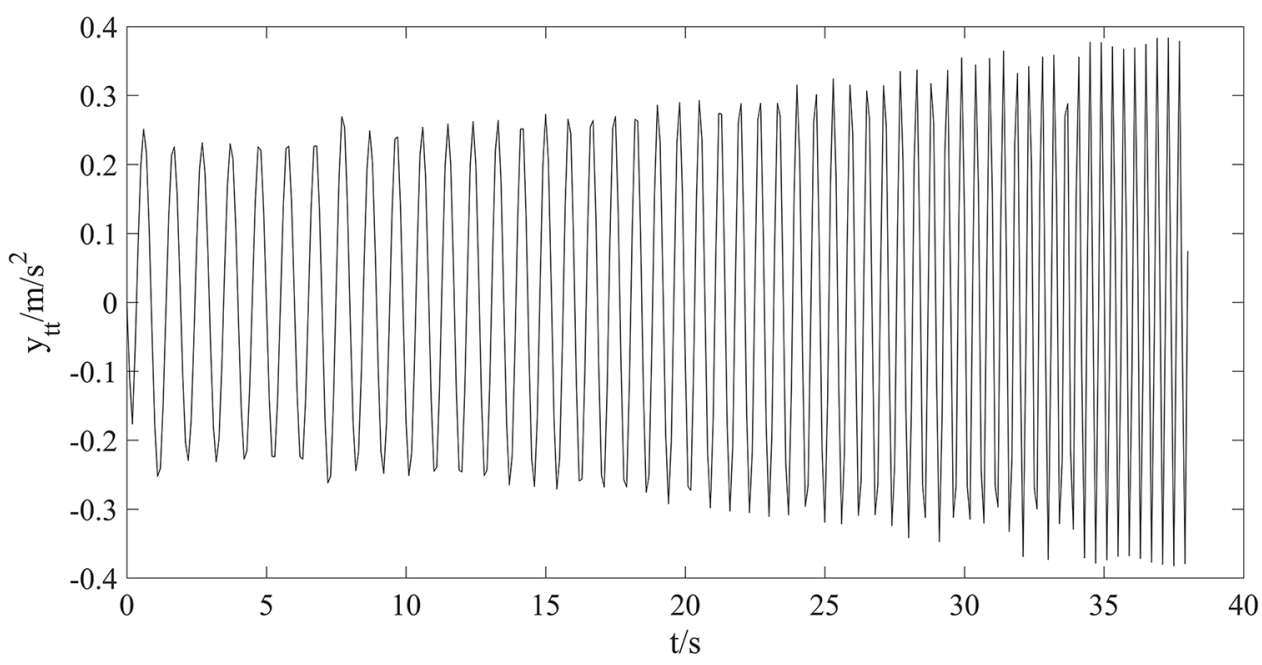

(a)

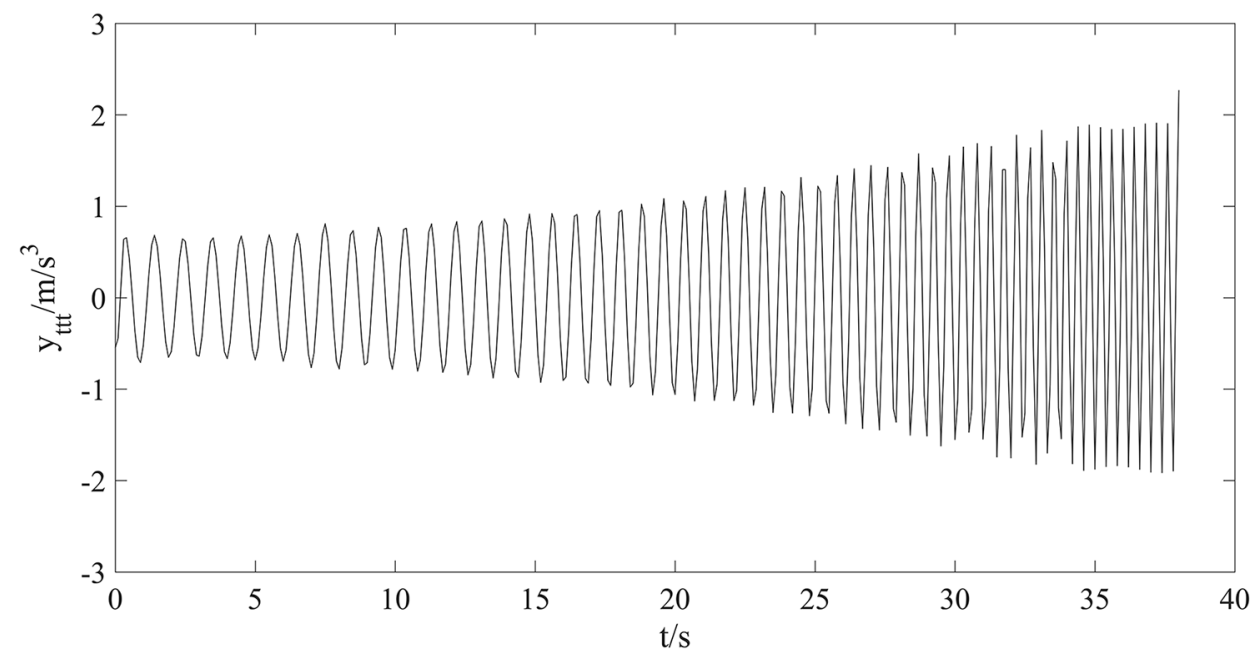

(b)

Fig. 4. Lifting system longitudinal acceleration and jerk response determination section. (a) Acceleration response (b) Jerk response.

Table 4. Acceleration response mean, standard deviation and coefficient of variation.

\begin{tabular}{llll}
\hline Time $(\mathrm{t} / \mathrm{s})$ & Mean $\left(\overline{\ddot{x}} /\left(\mathrm{m} / \mathrm{s}^{2}\right)\right)$ & Standard deviation $\left(\sigma_{\overline{\ddot{x}}} /\left(\mathrm{m} / \mathrm{s}^{2}\right)\right)$ & Coefficient of variation $(\mathrm{CV})$ \\
\hline 33 & -0.0748 & 0.0017 & 0.023 \\
33.1 & -0.3518 & 0.0099 & 0.028 \\
33.2 & -0.0072 & 0.0012 & 0.17 \\
33.3 & 0.3486 & 0.0015 & 0.004 \\
33.4 & 0.0804 & 0.0011 & 0.013 \\
33.5 & -0.3337 & 0.0060 & 0.017 \\
\hline
\end{tabular}

\subsection{Longitudinal acceleration and passenger comfort analysis of HELS}

Select $33-33.5 \mathrm{~s}$ with the largest vibration acceleration as the research object of acceleration response, determine the response $\ddot{q}_{d}$ as the acceleration response mean $\ddot{q}$. Calculate the standard deviation $\sigma_{\ddot{x}}$ due to the randomness of the parameters by combining (30) and Precise integration method for vertical vibration model of the time-varying system in the high-speed traction elevator, and calculate the coefficient of variation CV, the results are shown in Table 4 .

As can be seen from the Table 4 , in the case where the coefficient of variation of the random parameter is 0.02 , the coefficient of variation of the longitudinal vibration 


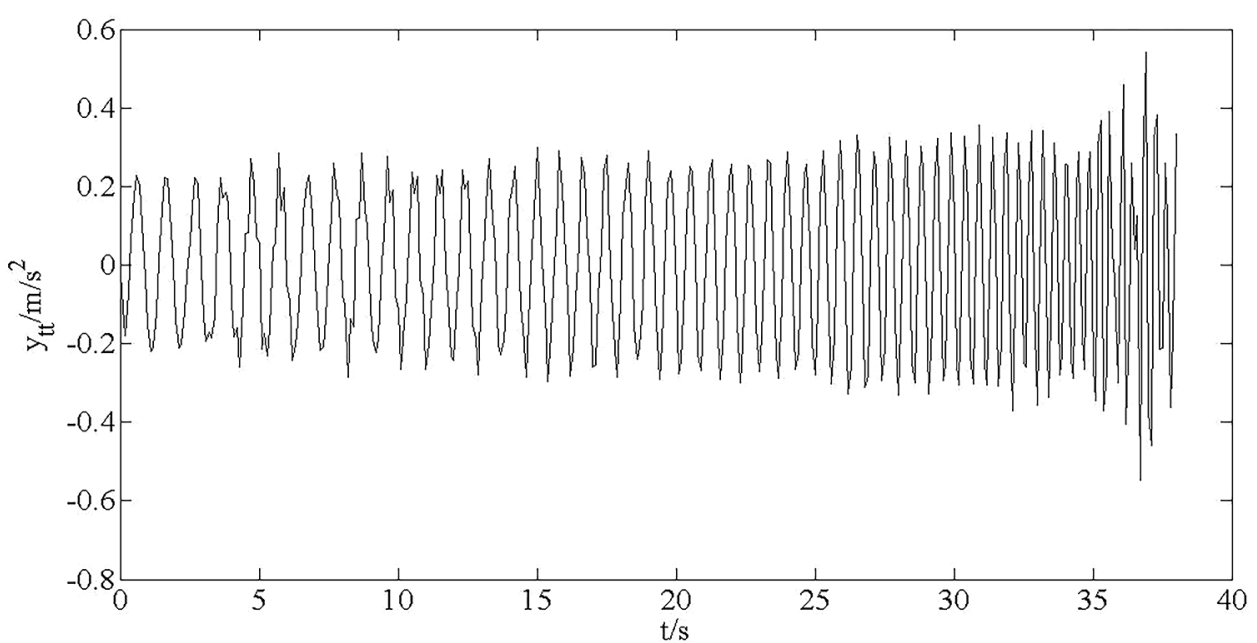

(a)

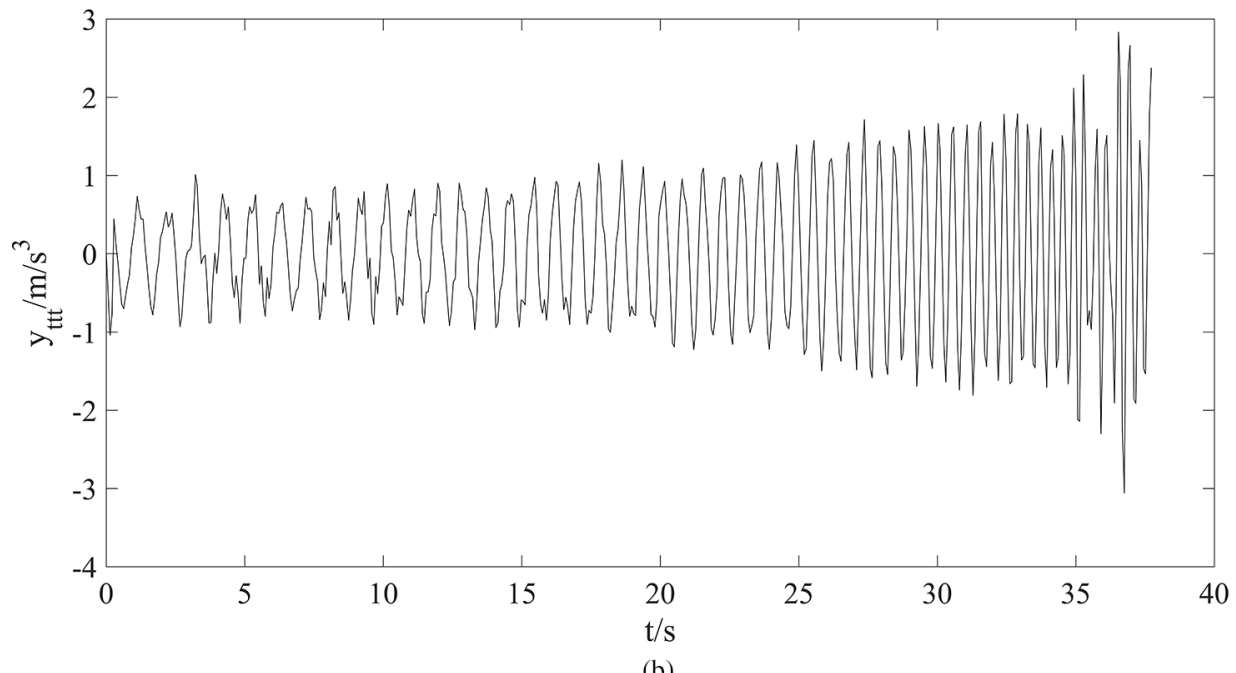

(b)

Fig. 5. Overall response of the longitudinal acceleration and jerk of the system. (a) Acceleration response (b) Jerk response.

acceleration response of high-speed elevators varies greatly. Compare the longitudinal vibration acceleration determination part and the total acceleration image obtained in the previous section, the actual response is more discrete, and the randomness of the system parameters is more obvious to the longitudinal vibration acceleration of high-speed elevators.

The whole process of car operation is selected as the research object, and the vibration does VDV is used to detect the passenger comfort [24]. VDV is defined as:

$$
V D V=\left[\int_{0}^{T} a_{w}^{4}(t) d t\right]^{1 / 4}
$$

where $\mathrm{T}$ is the duration of the vibration signal and $a_{w}^{4}$ is the acceleration the vibration signal after the weighting of the frequency meter. The VDV values of acceleration
Table 5. The VDV value of the determination part and total acceleration.

\begin{tabular}{lll}
\hline & VDV & Growth \\
\hline The determination part & 0.5078 & \\
The total acceleration & 0.5317 & 0.0239 \\
\hline
\end{tabular}

determination part and total acceleration of high-speed elevator lifting system are calculated respectively, as shown in Table 5.

As can be seen from the Table 5 , the VDV values of both are more than 0.5. Compared with the acceleration determination part of high-speed elevator lifting system, the VDV value increases by $4.71 \%$ after considering the influence of random parameters, which indicates that the influence of random parameters reduce the comfort of passengers. 


\section{Conclusion}

- In this paper, considering the mass of the compensation rope and the tension provided by the tensioning system, based on the axial string theory, combined with the energy method and the Hamilton principle, the longitudinal vibration time-varying continuous model of the HELS is constructed. The Galerkin method is used to transform the infinite dimensional partial differential equation into the ordinary differential equation with finite DOF. The fifth-order polynomial is used to fit the actual operating state parameters of the high-speed elevator and input as parameters of the dynamic equation. The precise integration method for longitudinal vibration model of the time-varying system in the high-speed traction elevatoris proposed, and the whole running process of elevator random dynamics is calculated.

- The determination and the random part response expression of the longitudinal dynamic response of the HELS are established by random perturbation theory. The displacement, velocity and acceleration sensitivity expressions of the random parameters are determined by solving the random response expression. The response sensitivity expression is used to solve the sensitivity values of each random parameter. It is found that the hoisting wire rope density has the highest sensitivity to longitudinal vibration velocity response, displacement response and acceleration response, the second is the lift mass, and the elastic modulus of the hoisting wire rope is the least sensitive. In the elevator manufacturing and installation process, parameters with strict sensitivity should be strictly controlled to improve the longitudinal dynamic performance of the high-speed elevator.

- Through the analysis of the digital characteristics of the acceleration response, the acceleration response and VDV values generated by the random parameters are calculated, which accurately reflects the dispersion degree of the longitudinal acceleration response and passenger comfort of the high-speed elevator under the influence of random parameters.

This research was supported by the Natural Science Foundation of Shandong Province (Grant No. ZR2017MEE049), the Introduce urgently needed talents project for the western economic uplift belt and the key areas of poverty alleviation and development in Shandong Province.

\section{References}

[1] W. Chen, Z. Ruijun, Z. Qing, Analysis of transverse vibration acceleration for a high-speed elevator with random parameter based on perturbation theory, Int. J. Acoustics Vibration 22, 218-223 (2017)

[2] B. Ji-Hu, Dynamics modeling and vibration control of high-speed elevator hoisting system, Shanghai Jiao Tong University, 2014
[3] C. Na, H. Wei, L. Zhi-Hao, Analysis of vertical vibration reduction strategy for elevator system, Noise Vibrat. Control 37, 117-137 (2017)

[4] W. Hui, Y. Wen-Hua, S. Yan, T. Zhi-Rong, Vertical vibration modeling and experimental analysis of high speed elevator, Machinery 51, 19-22 (2013)

[5] L. Zhi-Hao, H. Wei, L. Zhe-Kun, Analysis and suppression of vertical vibration in mechanical system of traction elevator, Machinery 55, 42-44 (2017)

[6] Y. Yan-Jie, High-speed traction elevator mechanical system vibration characteristic research, Shandong Jianzhu University, Shandong, 2015

[7] W. Li-Mei, G. Yu-Yan, L. Xue-Feng, Dynamic characteristics analysis of vertical vibration of haulage type elevator mechanical system, Mach. Des Manuf. 10, 16-18 (2007)

[8] S. Xin-Xin, Research on reliability sensitivity for the cabin and transmissionmechanism of elevators based on random parameters, Shandong Jianzhu University, Shandong, 2014

[9] Z. Rui-Jun, S. Xin-Xin, Y. Wei-Wei, D. Ming-Xiao, Resonance reliability sensitivity for a high-speeding elevator cabin system with random parameters, J. Vibrat. Shock 34, 84-88 (2016)

[10] Z. Qing, Y. Yu-Hu, Analysis of transverse vibration acceleration of a high-speed elevator with random parameters under random excitation, Period. Polytech. Mech. Eng. 61, 153-160 (2017)

[11] L. Yao, L. Yanbin, W. Hui, H. Yaozhi, Dynamic performance analysis and kinetic parameters optimization of high-speed elevator based on VPT, J. Vibration, Measurement \& Diagnosis. 35, 150-155 (2015)

[12] F. Wen-Zhou, C. Shu-Qian, Z. Feng, H. cheng, L. Wen-Bo, Resonance failure sensitivity for elevator system, J. Vibration and Shock. 34, 165-170 (2015)

[13] Z. Peng, Z. Chang-Ming, Analyses of longitudinal vibration and energetics on flexible hoisting systems with arbitrarily varying length, J. Shanghai Jiao Tong Univ. 42, 480-488 (2008)

[14] B. Ji-Hu, Z. Peng, Z. Chang-Ming, Transverse vibration of flexible hoisting rope with time-varying length, J. Mech. Sci. Technol. 28, 457-466 (2014)

[15] B. Ji-Hu, Z. Peng, Z. Chang-Ming, Dynamic analysis of flexible hoisting rope with time-varying length, Int. Appl. Mech. 51, 710-720 (2015)

[16] S. Hai-Ning, Y. Ya-Ping, Investigation on the precise integration method in structural dynamics, J. Qinghai Univ. 25, 6-10 (2007)

[17] D. Xiao-Qiang, M. De-Qing, C. Zi-Chen, Time-varying element model of high-speed traction elevator and its horizontal vibration response analysi, J. Zhejiang Univ. 43 148-152 (2009)

[18] D. Zi-Chen, Z. Huan-Jun, Z. Yu-Li, Z. Wan-Xie, On computation of dynamic properties for deploying cantilever beam based on precision integration method, J. Astron. 22 110-113, (2001)

[19] Z. Wan-Xie, Precise computation for transient analysis, Chin. J. Comput. Mech. 25, 1-6 (1995)

[20] W. Shi-Long, T. Bo, Z. Yu, Z. Jie, C. Cheng, Improved shock load model of stranded sireshelical springs based on perturbation method, J. Mech. Eng. 51, 85-90, (2015) 
[21] H. Gong-Yu, L. Jing-Jing, Z. Wei-Weietc, Perturbation solutions for elasto-plastic problems of circular tunnel under unequal compression, Chin. J. Rock Mech. Eng. 33, 3639-3647 (2014)

[22] N. Ming-Tao, L. Chang-Sheng, C. Li-Yuan, Perturbation methods for structural-acoustic coupled systems with interval parameters, J. Vib. Shock 34, 194-198 (2015)
[23] Z. Yi-Min, L. Qiao-Ling, W. Chun-Bang, Probability perturbation finite element method for response analysis of multi-degree-of-freedom nonlinear vibration systems with random parameters, Chin. J. Comput. Mech. 20, 8-11 (2003)

[24] British Standard Guide, BS 6841: Measurement and evaluation of human exposure to whole-body mechanical vibration and repeated shock, BSI, London, 1987

Cite this article as: Q. Zhang, T. Hou, H. Jing, R. Zhang, Analysis of longitudinal vibration acceleration based on continuous time-varying model of high-speed elevator lifting system with random parameters, Mechanics \& Industry 22, 28 (2021) 